\title{
Spectroscopy and Photoelectrochemistry of Organic Monolayers within Sphere-Plane Gold Nano-Gaps
}

\author{
Katsuyoshi IKEDA \\ Division of Chemistry, Graduate School of Science, Hokkaido University (Sapporo 060-0810, Japan)
}

Received April 8, 2011 ; Accepted May 16, 2011

\begin{abstract}
Plasmonic field localization is normally utilized on irregularly roughened surfaces or nanostructured surfaces of coinage metals. Although localized fields increase photon-molecule interactions, metal-molecule interactions become an uncontrollable variable because atomic surface features are not organized in such nanostructured metals. However, construction of sphere-plane nano-gaps on atomically defined metal surface enables us to manage metal-molecular interactions in addition to control of plasmonic resonance features. In SERS spectroscopy using this system, well-managed electromagnetic and chemical contributions provide detailed information on geometric and electronic structures of molecular adsorbates. Moreover, highly localized fields within the nano-gaps extend the range of SERS application to highly damping catalytic metal surfaces. In photo-energy conversion, incident photon to current conversion efficiency in porphyrin-based molecular monolayer system is largely increased by optical antenna effect of the nano-gaps.
\end{abstract}

Key Words : Plasmon Resonances, SERS, Photo-Eenergy Conversion, Single Crystal Surface

\section{Introduction}

In the fields of spectroscopy, photochemistry, and photoelectrochemistry, one of main issues is to achieve high efficiency of photon-molecule interactions. Unfortunately, photons and molecules interact rather weakly because of dimensional difference between photon field distribution on subwavelength (submicrometric) scale and molecular size on nanometric scale. Therefore, spatial localization of photon fields should increase photon-molecule coupling. To localize photon fields beyond the diffraction limit, a promising method is to use plasmon resonances on a metal surface, which are collective oscillation of free electrons. ${ }^{1)}$ When molecules are placed within such highly localized fields, the interaction efficiency is expected to be increased. In this sense, metal nanostructures are considered to be "nano-light sources" or "optical antennae".

Plasmon resonances are quite sensitive to shape and size of metal structures. To increase the degree of field localization, therefore, various metal nanostructures have been designed on the basis of the electromagnetic theory. However, such optical engineering does not take into account physical and chemical interactions between molecules and metal nanostructures. Actually, metal-molecule interactions are strongly affected by atomic surface features of nanostructures; for example, catalytic and electrocatalytic reactions are often crystal-face dependent. ${ }^{3)}$ When plasmonic field-localization is applied to a molecule-based photochemical system, therefore, it is indispensable to control both plasmonic structures on nanometric scale and surface features on atomic scale. According to the recent progress in nanotechnology, plasmonic structures are now becoming controllable by lithographic top-down fabrication methods. However, it is still difficult to manage atomic surface features of plasmonic structures.

In this review, we demonstrate that a sphere-plane type plasmonic structure has a great advantage for controlling both photon-molecule interactions and metalmolecule interactions, based on our recent publications. ${ }^{4-9)}$ Application of this structure enables us to observe molecular adsorbates on a well-defined single crystalline electrode surface by surface enhanced Raman scattering (SERS) and to increase photo-energy conversion efficiency in a molecular monolayer system.

\section{Plasmonic Structure with a Well-defined Metal-molecular Interface}

To control metal-molecular interfaces in a plasmonic system, it is indispensable to manage atomic surface arrangements of a metal nanostructure. One of possible approach organizing a nanostructured surface is to construct plasmonic structures onto a well-defined single crystalline metal surface. Figure 1a shows a schematic illustration of a sphere-plane type plasmonic structure, which consists of atomically defined single crystal metal electrode, highly ordered organic monolayer, and metal nano-sphere. This structure can be practically constructed on a monolayer-modified metal electrode by physisorption of a metal nanoparticle.) Although this bottomup construction is rather simple, the chemisorbed interface between the monolayer and the electrode is well-organized on atomic or molecular scale.

Plasmon resonance features of the structure have already been theoretically studied by Metiu et al. ${ }^{10,11)} \mathrm{In}$ the absence of the sphere, surface plasmons cannot be excited at planar surface because wave vectors of surface plasmons do not match with those of photons. However, when a metal nanosphere is nearly in touch with a metal plane, highly localized electromagnetic fields can 
be excited even at atomically smooth surface. This is because dipole-active bonding plasmons, so-called gapmode plasmons, are created by hybridization between the plasmon of the sphere and that of the planar surface. This situation can be understood as follows (see Fig. 1 (b)): the incident light field polarizes the sphere. This polarized field corresponds to dipolar plasmons $(l=1)$. When the sphere is placed close to the surface, it is polarized by its image field in addition to the polarization by the incident field. Since the image field is spatially inhomogeneous, it can excite multipolar plasmons $(l=2,3$ ...) in the sphere. As a result of the electromagnetic interactions between the sphere and the substrate, hybridized bonding gap-mode plasmon modes are built, resulting in substantial enhancement of the electric fields within the gap between the sphere and the substrate. This means photon-molecule interactions are largely increased in this spatial region. In this structure, the plasmon resonance feature strongly depends on separation between sphere and plane. This can be seen extinction spectra of Au-nanoparticle (Au-NP) with a diameter of $20 \mathrm{~nm}$ above Au planar substrate under a given space, which are theoretically calculated by Wind's method (Fig. 2). ${ }^{12,13)}$ The plasmon resonance peak is red shifted with decreasing the distance, indicating that precise distance control between sphere and plane is required for management of field localization. Experimentally, the gap distance is well controlled by the monolayer thickness on an atomically smooth single crystal surface, and
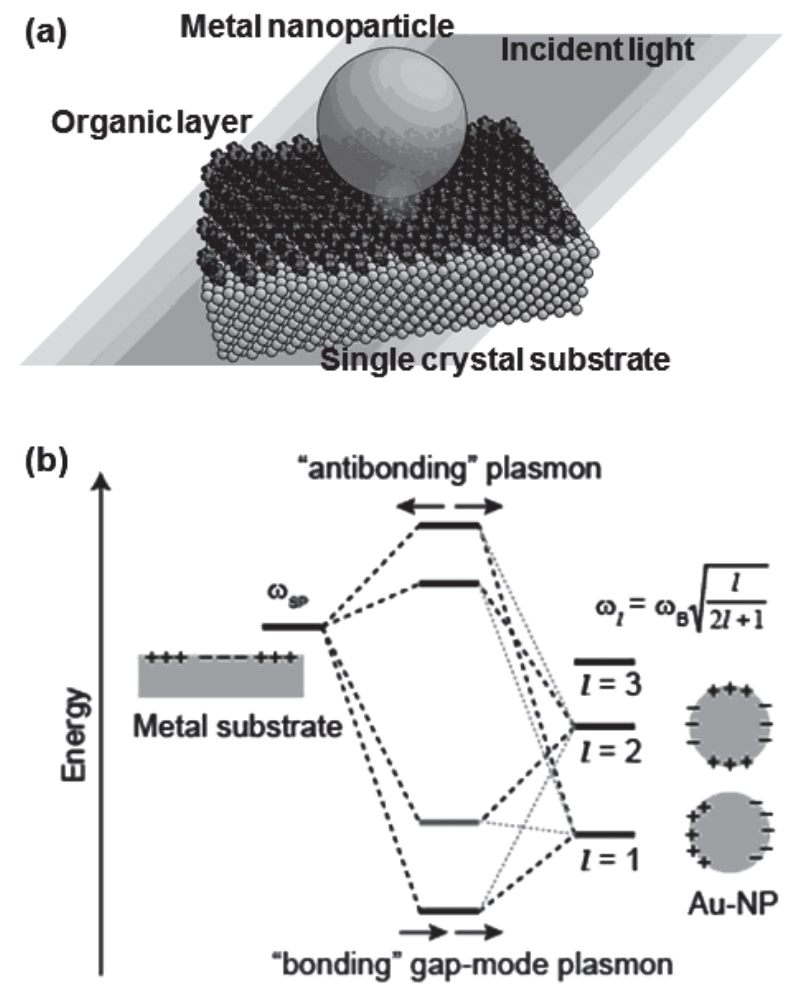

Fig. 1 (a) Schematic illustration of a sphere-plane gold nano-gap, which consists of atomically defined single crystal metal electrode, highly ordered organic monolayer, and metal nano-sphere. (b) Energy diagram for plasmon hybridization between sphere and substrate. ${ }^{8)}$

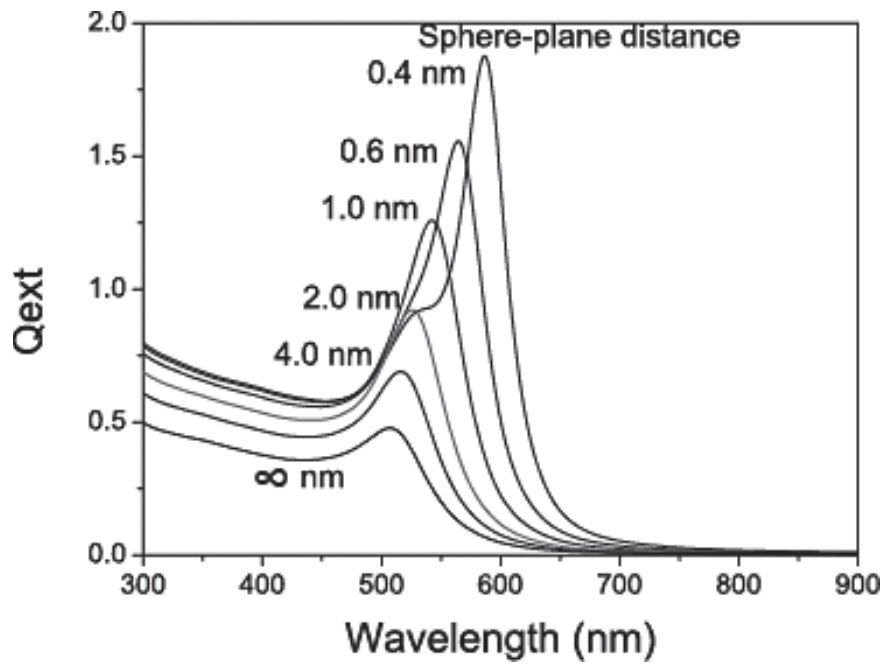

Fig. 2 Extinction spectra of Au sphere with a diameter of $20 \mathrm{~nm}$ above Au planar substrate under a given space, which were theoretically calculated by Wind's method. ${ }^{8)}$

hence, highly reproducible plasmon resonances are available when mono-dispersed metal nanoparticles are utilized to form a number of the structures on an electrode surface.

Incidentally, the attenuated total reflection (ATR) method in Otto configuration might be also considerable for plasmonic enhancement at a planar metal surface. ${ }^{14)}$ However, the degree of field localization is rather low and barely controllable in this system. A tip-enhancement method such as Tip-enhanced Raman spectroscopy (TERS) also enables us to increase photon-molecule interactions at a smooth metal surface, ${ }^{15)}$ but it is still technically difficult especially in electrochemical conditions.

\section{SERS Spectroscopy at a Well-defined Metal Surface}

Vibrational spectroscopy is a powerful experimental method to obtain molecular level information. Among various spectroscopic techniques, SERS can in principle be a powerful way to study electrochemical reactions especially in IR-opaque electrolyte solution because of its high sensitivity to molecular adsorbates on a metal surface $^{16,17)}$ In practice, however, application of SERS is strictly limited to roughened surfaces of coinage metals such as $\mathrm{Au}, \mathrm{Ag}$, and $\mathrm{Cu}$. This limitation severely reduces the range of application of SERS. That is, SERS is not useful to observing a well-defined smooth metal surface and is not applicable to catalytic metals such as Pt or Pd even on a roughened surface. Enhancement factor (EF) in SERS can be described as follows: ${ }^{18)}$

$$
E F=\left|\alpha_{\mathrm{R}} / \alpha_{\mathrm{R} 0}\right|^{2}\left|g\left(\omega_{\mathrm{sc}}\right) g\left(\omega_{\mathrm{i}}\right)\right|^{2},
$$

where $g\left(\omega_{\mathrm{sc}}\right)$ and $g\left(\omega_{\mathrm{i}}\right)$ indicate local field enhancement on metal surface at scattered light frequency $\omega_{\text {sc }}$ and incident light frequency $\omega_{\mathrm{i}}$, respectively. $\alpha_{\mathrm{R}}$ and $\alpha_{\mathrm{R} 0}$ are the Raman polarizabilities of molecules with and without adsorption on metal surface. Electromagnetic (EM) contributions to SERS are ascribed to the $\left|g\left(\omega_{\mathrm{sc}}\right) g\left(\omega_{\mathrm{i}}\right)\right|^{2}$ 
term in this equation. To increase the magnitude of this term, an electrochemical procedure for surface roughening is normally conducted in conventional SERS spectroscopy. Conversely, metal-molecule interactions contribute to SERS via the $\left|\alpha_{R} / \alpha_{R 0}\right|^{2}$ term. This chemical (CM) contribution is sensitive to atomic surface features. In conventional SERS measurements on irregularly roughened metal surface, therefore, there are many uncontrollable variables in both of EM and CM contributions, resulting in the difficulty in quantitative and reproducible spectroscopic investigations on metal-organic interactions.

When sphere-plane type plasmonic structures are applied to SERS measurements, both contributions can be well-managed, leading to a possibility of disentangling these two contributions to SERS. ${ }^{8)}$ Figure 3 shows SERS spectra of p-aminothiophenol (pATP) monolayer on defined $\mathrm{Au}(111)$ and $\mathrm{Au}(100)$ faces without and with sphere-plane nano-gaps. In the absence of the nano-gaps, no Raman signal was detected at both faces because surface plasmons could not be excited at such a smooth surface. Conversely, when Au-NPs with diameter of $c a .20$ $\mathrm{nm}$ were deposited on the molecular layers, the signal intensities were substantially enhanced. EF for $\mathrm{Au}(100)$ was estimated to be the order of $10^{5}$ with respect to the $7 \mathrm{a}(\mathrm{a} 1)$-intensity. On the other hand, EF for the $\mathrm{Au}(111)$ was fourfold larger than that for $\mathrm{Au}(100)$. Furthermore, the ratio of b2 to a1 was significantly different in these spectra; for example, the ratio of $9 \mathrm{~b}(\mathrm{~b} 2)$ to $7 \mathrm{a}(\mathrm{a} 1)$ is 0.43

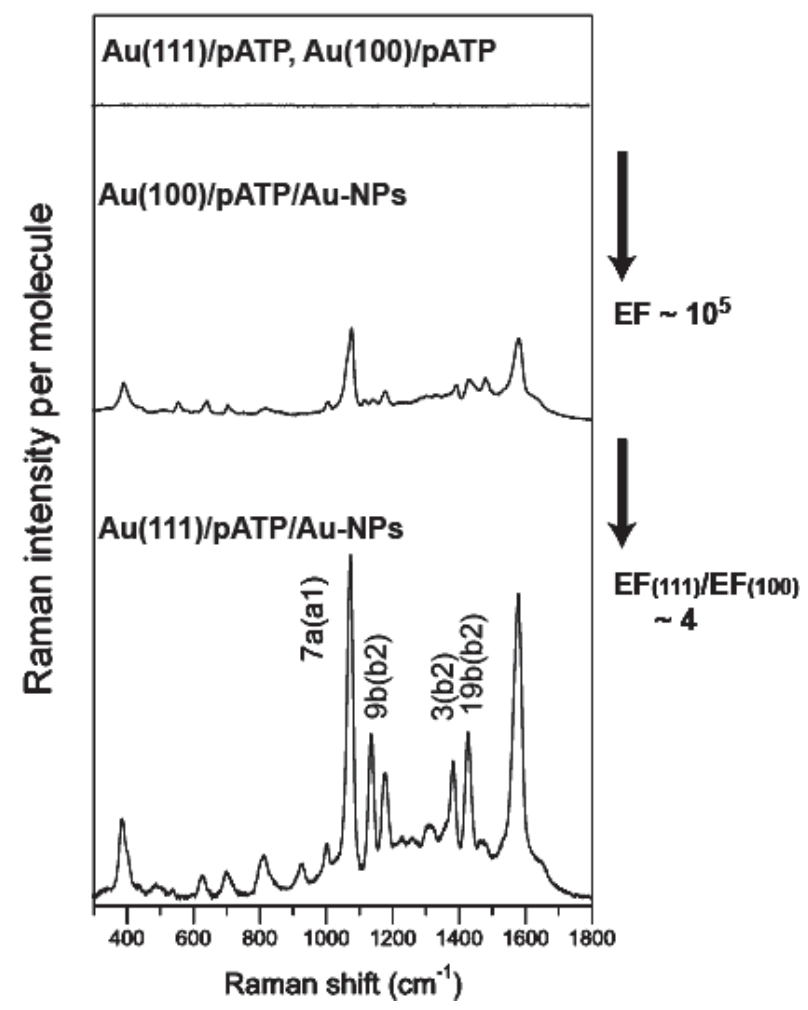

Fig. 3 SERS spectra of p-aminothiophenol (pATP) monolayer on defined $\mathrm{Au}(111)$ and $\mathrm{Au}(100)$ faces without and with sphere-plane plasmonic cavities, excited by $633-\mathrm{nm}$ He-Ne laser radiation. ${ }^{8)}$ for $\mathrm{Au}(111)$ and 0.06 for $\mathrm{Au}(100)$. Note that the EM contributions to the EF should be comparable between the different facets. This is because the plasmonic property of the sphere-plane nano-gap, which is characterized by plasma frequency of the bulk metal, is independent of crystal orientations. Consequently, it is naturally concluded that the crystal face dependence of the EFs must be ascribed to the difference of the degree of $\mathrm{CM}$ contributions. More specifically, the extra intensity gain on $\mathrm{Au}(111)$ can be explained by Franck-Condon A-term contribution for al modes and by the Hertzberg-Teller type vibronic C-term for b2 modes. ${ }^{18)}$ Such crystal face dependent information is not expected in conventional SERS.

The other problem in conventional SERS is that signal enhancement is only strong on coinage metals such as $\mathrm{Au}, \mathrm{Ag}$, and $\mathrm{Cu}$. Most of other metals, including catalytic metals such as Pt or Pd, are commonly considered to be non-SERS-active. ${ }^{19,20)}$ This is because surface plasmons are not efficiently exited on such a highly damping metal surface. Importantly, sphere-plane type plasmonic structures are effective even for highly damping metals because of their extremely high field localization. ${ }^{5.7)}$

Figure 4 (a) shows conventional SERS spectra of 4-chlorophenyiisocianide (CPI) monolayers on electrochemically roughened surfaces of $\mathrm{Au}, \mathrm{Pt}$, and $\mathrm{Pd}$. On roughened $\mathrm{Au}$, Raman active modes of CPI were clearly observed in the spectrum; the NC stretching frequency (vNC) of $2180 \mathrm{~cm}^{-1}$ indicates bond formation between anchor group of CPI and Au substrate. In the case of roughened $\mathrm{Pt}$ and $\mathrm{Pd}$, however, SERS activity was quite low and the distinct Raman bands were somehow obtained only on Pt. The $v \mathrm{NC}$ frequency of $2144 \mathrm{~cm}^{-1}$ on Pt was slightly lower than that on $\mathrm{Au}$, indicating that the degree of back donation from metal to CPI is dependent on substrate metals. Moreover, there appeared additional peak at $1565 \mathrm{~cm}^{-1}$ in addition to the totally symmetric $v \mathrm{C}=\mathrm{C}$ (8a) mode at $1586 \mathrm{~cm}^{-1}$ on $\mathrm{Pt}$, which will be discussed later.

Figure 4 (b) shows sphere-plane cavity-induced SERS (a)

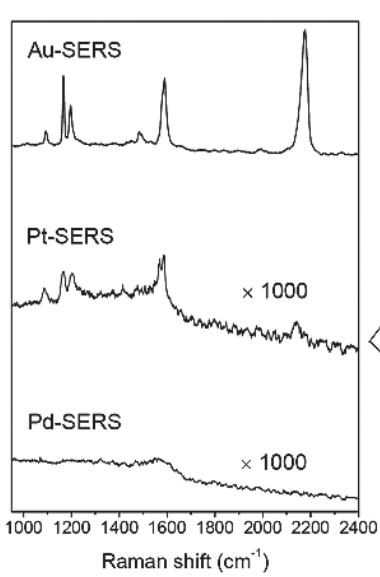

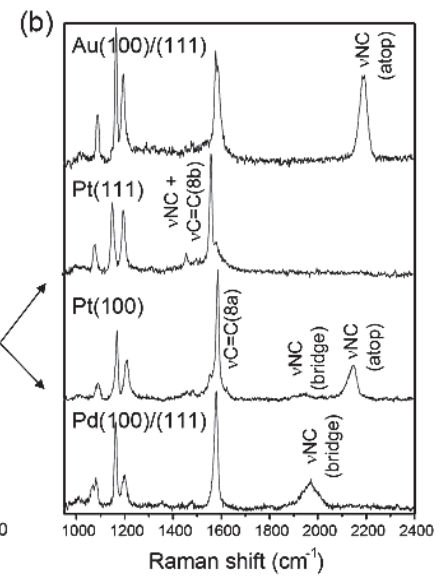

(b)

Raman shift $\left(\mathrm{cm}^{-1}\right)$
Fig. 4 (a) Conventional SERS spectra of 4-chlorophenyiisocianide (CPI) monolayers on electrochemically roughened surfaces of Au, Pt, and Pd. (b) Sphere-plane cavity-induced SERS spectra of CPI monolayers on defined (111) and (100) faces of $\mathrm{Au}, \mathrm{Pt}$, and Pd. 
spectra of CPI monolayers on defined (111) and (100) faces of $\mathrm{Au}, \mathrm{Pt}$, and $\mathrm{Pd}$. One can see that substantial enhancement of Raman scattering signals was obtained on all of the metals including "non-SERS-active" Pt and Pd. EFs for Pt and Pd were estimated to be the order of $10^{5}$, which is 1000 times larger than previously reported value. ${ }^{5)}$ Moreover, the SERS spectra on Pt clearly showed crystal face dependence. Especially, the spectral feature of CPI/Pt(111) was largely different from all others; there was no $v \mathrm{NC}$ peak at $2144 \mathrm{~cm}^{-1}$ and no $8 \mathrm{a}$ peak at $1586 \mathrm{~cm}^{-1}$. According to the theoretical vibrational calculations, the adsorption geometry of CPI was determined to be atop at $\mathrm{Au}(111) /(100)$, bridge at $\operatorname{Pd}(111) /(100)$, and coexistence of atop and bridge at $\mathrm{Pt}(100)$ and 3-fold hollow at Pt(111). Note that the conventional Pt-SERS spectrum can be reproduced as a sum of $\mathrm{Pt}(111)$ - and $\mathrm{Pt}(100)$ spectra, suggesting that conventional SERS spectra appear as a combination of contributions from multiple crystalline faces.

\section{Plasmonic Enhancement in Photo-energy Conversion}

In molecular based photo-energy conversion system, electronic excited states of dye molecules are utilized to promote uphill electron transfer. ${ }^{21)}$ One of key issues in such photo-driven molecular system is to increase incident photon to current conversion efficiency (IPCE). Application of plasmonic metal nanostructures should increase photon-molecule interactions and thus increase the population of the excited molecules. However, the presence of the plasmonic structures decrease the lifetime of the excited states because of energy transfer from excited dye molecules to the metals, resulting in lowering of internal quantum efficiency. This problem is an unavoidable serious problem in plasmonic enhancement of photo-energy conversion. EF can be described as:

$$
E F=\left(\eta / \eta_{0}\right)\left|g\left(\omega_{\mathrm{i}}\right)\right|^{2},
$$

where $\eta$ and $\eta_{0}$ are the internal quantum efficiency of photo-induced electron transfer with and without plasmonic nanostructures. EM contribution to photo-energy conversion is only proportional to the square of $g$ whilst that to SERS to the fourth-power of $g$. Besides, the $\eta$ value is normally smaller than the $\eta_{0}$ value. Therefore, it is essential to maximize field localization and to minimize lowering of the quantum efficiency simultaneously. However, precise control of these competitive effects is not easy in conventional plasmonic system. Both effects are sensitive to distance between metal and dye molecules, and are largest when the molecule is very close to the surface. On the other hand, in the case of the sphereplane type structure, EF is not as strongly dependent on the spatial position within the gap. Therefore, one of possible approach is to construct sphere-plane type plasmonic structures on a well-defined smooth electrode surface and to locate dye molecules at mid-distance between sphere and plane. ${ }^{11)}$

To demonstrate plasmonic enhancement of photo-ener- gy conversion, self-assembled monolayers were formed on atomically smooth $\mathrm{Au}$ electrode surface using porphyrin-ferrocene-thiol linked molecules. ${ }^{21)}$ In this molecular system, excitation of porphyrin induces cathodic photocurrent from $\mathrm{Au}$ electrode to electron acceptors in solution. Figure 5 (a) shows photo-current action spectra before and after formation of nano-gaps on the monolayer covered electrode. ${ }^{9)}$ In this experiment, $50 \mathrm{~nm}$ Au-NPs were utilized as a part of the nano-gaps. One can see that the presence of Au-NPs largely enhanced IPCE in the entire visible wavelength region, suggesting optical antenna effect of gold nano-gaps. Note that surface density of porphyrin does not change during the procedure of the nano-gap formation. Therefore, $\mathrm{EF}$ is equal to the ratio of photocurrent with and without Au-NPs. As shown in Fig. 5 (b), the photocurrent was enhanced by a factor of 20 around $670 \mathrm{~nm}$. From similarity of wavelength dependence between experimentally obtained $\mathrm{EF}$ and theoretically calculated extinction of the sphereplane system, one can conclude that the enhancement of photocurrent is indeed caused by plasmonic field localization. Incidentally, the observed EF is thought to be averaged for the entire surface area of the electrode since our optical antenna effect is spatially inhomogeneous. According to the surface coverage with Au-NPs

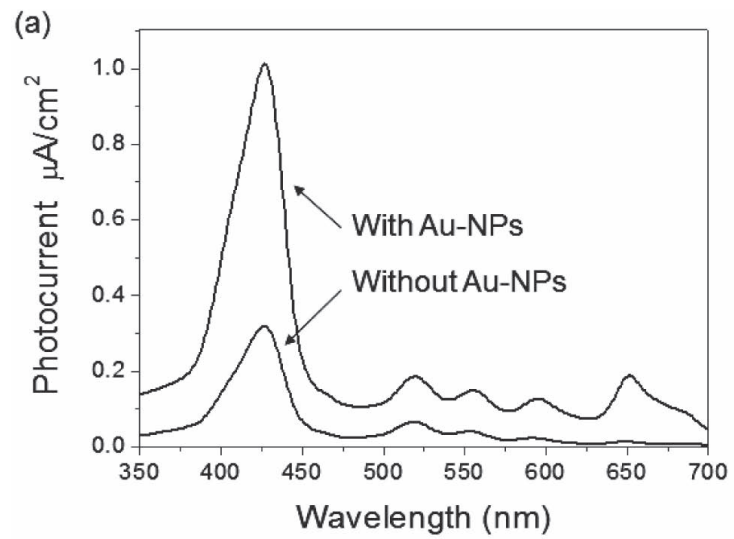

(b)

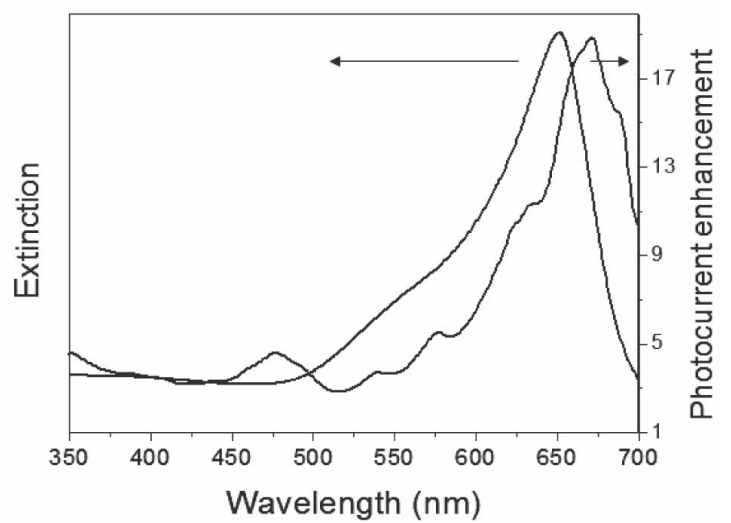

Fig. 5 (a) Photo-current action spectra of porphyrin-based molecular monolayers on atomically smooth Au electrode before and after adsorption of Au-NPs with diameter of 50 nm. (b) Excitation wavelength dependence of efficiency enhancement measured in photo-induced uphill electron transfer and calculated extinction spectrum of the gold nano-gap. ${ }^{9)}$ 
obtained from SEM photographs, the intrinsic enhancement in each "hot spot" can be estimated to be more than 50 .

The present result shows that sphere-plane type nanostructures are favorable as optical antennas for photodriven molecular monolayer system. Although fabrication procedure is rather simple, the antenna property is in principle tunable by changing sphere diameter and photon energy is effectively confined into photo-sensitive molecular layer located within sphere-plane gaps.

\section{Conclusion}

Control of atomic surface features in plasmonic metal nanostructures is indispensable to realize well-managed photon-molecule interactions. We demonstrated that the sphere-plane type plasmonic structure is one of simple solutions to this problem. Although plasmonic structures are normally combined with molecular system by forming of organic layer onto nanostructured metal electrode, our system is completely opposite; metal nanoparticles are deposited onto molecular-modified smooth metal electrode so that metal nano-gaps are formed. This bottom-up approach is effective to control both plasmonic resonance properties and metal-molecular junctions, and is widely applicable to various molecular-functionalized electrodes.

By using this plasmonic system in SERS spectroscopy, spectral reproducibility is substantially enhanced via well-managed EM and CM contributions. The range of application of SERS is significantly extended to atomically defined surfaces of various metals including highly damping catalytic metals. We have indeed confirmed crystal face dependence of CM contributions to SERS as well as crystal face dependent adsorption geometry of molecules. This system can be utilized even in electrochemical conditions. ${ }^{8)}$ In principle, SERS spectroscopy has a great advantage for probing low-frequency vibrational modes such as molecule-substrate stretching. Insitu SERS observations should be useful for studying reaction mechanisms of electrocatalysts. The present method would improve our knowledge about catalytic reactions and electrochemical reactions.

Application of plasmonic field localization to the photofunctionalized molecular system also increased IPCE in visible wavelength region, even though the presence of metal nanostructures may lower the internal quantum efficiency. The sphere-plane type nano-gaps are suitable for use as optical antennas in ultrathin organic monolayers. This remarkable feature could lead to the use of this technique in molecular electronic devices. This strategy should open new possibilities in the design of molecular devices.

\section{Acknowledgments}

This research was supported by Grant-in-Aid Scientif- ic Research on Priority Area "Strong Photon-Molecule Coupling Fields" (21020001), Grant-in-Aid for Young Scientists (B) (22750001), World Premier International Research Center (WPI) Initiative on Materials Nanoarchitechtonics, Global COE program (Project No. B01: Catalysis as the Basis for Innovation in Materials Science), and MEXT Program for Development of Environmental Technology using Nanotechnology from Ministry of Education, Culture, Sports, Science and Technology, Japan. Au-NPs used for photo-energy conversion experiments were provided by Dr. T. Teranishi and Dr. M. Kanehara (University of Tsukuba). The author also acknowledges valuable discussions with Prof. K. Uosaki (Hokkaido University).

\section{References}

1) W. L. Barnes, A. Dereux, and T. W. Ebbesen, Nature, 424, 824 (2003).

2) P. Bharadwaj, B. Deutsch, and L. Novotny, Adv. Opt. Photon., 1, 440 (2009).

3) N. M. Marković and P. N. Ross Jr., Surf. Sci. Rep., 45, 117 (2002).

4) K. Ikeda, N. Fujimoto, H. Uehara, and K. Uosaki, Chem. Phys. Lett., 460, 205 (2008).

5) K. Ikeda, J. Sato, N. Fujimoto, N. Hayazawa, S. Kawata, and K. Uosaki, J. Phys. Chem. C, 113, 11816 (2009).

6) U. Jung, M. Müller, N. Fujimoto, K. Ikeda, K. Uosaki, U. Cornelissen, T. Felix, C. Bornholdt, D. Zargarani, R. Herges, and O. Magnussen, J. Colloid Interface Sci., 341, 366 (2010).

7) K. Ikeda, J. Sato, and K. Uosaki, J. Photochem. Photobio. A: Chem., 221, 175 (2011).

8) K. Ikeda, S. Suzuki, and K. Uosaki, Nano Lett., 11, 1716 (2011).

9) K. Ikeda, K. Takahashi, T. Masuda, and K. Uosaki, Angew. Chem. Int. Ed., 50, 1280 (2011).

10) P. K. Aravind and H. Metiu, J. Phys. Chem., 86, 5076 (1982).

11) P. K. Aravind and H. Metiu, Surf. Sci., 124, 506 (1983).

12) M. M. Wind, J. Vlieger, and D. Bedeaux, Physica A, 141, 33 (1987).

13) T. Okamoto and I. Yamaguchi, J. Phys. Chem. B, 107, 10321 (2003).

14) A. Otto, Z. Phys., 216, 398 (1968).

15) B. Pettinger, B. Ren, G. Picardi, R. Schuster, and G. Ertl, Phys. Rev. Lett., 92, 096101 (2004).

16) A. Otto, I. Mrozek, H. Grabhorn, and W. Akemann, J. Phys.: Condens. Matter, 4, 1143 (1992).

17) M. Moskovits, Rev. Mod. Phys., 57, 783 (1985).

18) J. R. Lombardi and R. L. Birke, J. Phys. Chem. C, 112, 5605 (2008).

19) M. Futamata, E. Keim, A. Bruckbauer, D. Schumacher, and A. Otto, Appl. Surf. Sci., 100/101, 60 (1996).

20) M. E. Abdelsalam, P. N. Bartlett, J. J. Baumberg, and A. E. Russell, J. Am. Chem. Soc., 129, 7399 (2007).

21) K. Uosaki, T. Kondo, Z.-Q. Zhang, and M. Yanagida, J. Am. Chem. Soc., 119, 8367 (1997). 